\title{
Characterization of rectal neoplasms using computerized tomography
}

\author{
Caroline Edward Ayad*, Wael Abdelgafour Mohammed, Elsafi Ahmed Abdalla, \\ Samih Awad Kajaok
}

Radiology Department, Sudan University of Science and Technology, Khartoum, Sudan

\section{Email address:}

carolineayad@yahoo.com, carolineayad@sustech.edu (C. E. Ayad), waelabdelgafour@yahoo.com (W. A. Mohammed), dr.elsafi@sustech.edu (E. A. Abdalla), Samihkajoak@yahoo.com (S. A. Kajoak)

\section{To cite this article:}

Caroline Edward Ayad, Wael Abdelgafour Mohammed, Elsafi Ahmed Abdalla, Samih Awad Kajaok. Characterization of Rectal Neoplasms Using Computerized Tomography. American Journal of Health Research. Vol. 2, No. 2, 2014, pp. 62-67.

doi: 10.11648/j.ajhr.20140202.15

\begin{abstract}
The aims of this study were to characterize and localize the rectal neoplasms by the computerized tomography (CT) and sigmoidoscopy in patients who suspected to have colorectal neoplasms. It was conducted at Radiology and Endoscopy Departments - Soba Hospital-Khartoum-Sudan. This study was extended from October 2011 to December 2011, fifty patients were studied including 34 males and 16 females, and their mean age was 52.5 years ranged from $25-85$ years. All patients underwent sigmoidoscopy, axial CT images for pelvis, enhanced with contrast medium, and biopsy as confirmation method. The location of rectal neoplasm was measured in $(\mathrm{cm})$ and the lesions were characterized in CT as circumferential, eccentric rectal wall thickening, masses, tumors according to thier contrast enhancement, in Sigmodioscopy the lesions were classified as seen into polyploidy, obstructive, ulcerative, circumferential lesions and tumor. In the histopathology findings, the lesions were characterized as poor, moderate, well differentiated adenocarcinoma and Tubulovillous adenoma.The study showed that the males were more affected than females. Sigmoidoscopy and CT can predict the lesion type and characterize the changes in the rectum and rectosigmoid region according to its citation from the anal verge significantly at $p$-value 0.001 and 0.038 . No significant relation was found between the biopsy results and the presence of the lesions through distance from anus at $P$-value 0.161 . When comparing the CT, sigmoidoscopy and biopsy results; CT has an accuracy of $72 \%$ and sensitivity of $69.9 \%$, sigmoidoscopy has an accuracy of $86.0 \%$ and sensitivity of $88.9 \%$ where the biopsy has accuracy of $90 \%$ and sensitivity $89.1 \%$ in differentiation of rectal neoplasms. It can be believed that $\mathrm{CT}$ and sigmoidiscopy should be performed together and used as essential methods in diagnosing of rectal neoplasms in addition to histopathological examination as an effective tool in determining the possibility of malignancy of this detected neoplasms.
\end{abstract}

Keywords: Rectal Neoplasm, CT, Sigmoidscopy

\section{Introduction}

Rectal cancer is a common cancer and a major cause of mortality in Western countries. The diagnosis includes rectal clinical examination, endoscopy, barium studies and histopathology, enhanced by biochemistry laboratory test. All of these techniques are poor indicators of the invasion and lymph node involvement, which are both important features for prediction of diagnoses [1-5].Studies showed that colonoscopy is the most sensitive method for the detection of colorectal cancer or adenomatous polyps [6] colonoscopy is highly sensitive; examines the entire colon; and allows for screening, diagnosis, and removal of polyps but, colonoscopy has a higher risk of complications than other investigation methods including bowel tears or bleeding .Double contrast Barium enema allows complete radiological examination of the colon .This method is less sensitive than colonoscopy for visualizing small polyps or cancer [7].Number of imaging methods is available including Trans rectal ultrasound (TRUS), Magnetic Resonance Imaging (MRI), Computed tomography (CT) scanning for diagnosis colorectal carcinoma. Ultrasonography enables to distinguish layers within the rectal wall; it is an accurate method for detecting depth of tumor penetration and perirectal spread [8]. Its sensitivity ranges between $83 \%$ and $97 \%[9,10]$ it has not been shown to 
be predictive of the histology of the visualized lymph nodes [11].MRI can predict a histologically involved circumferential margin with sensitivity of $94 \%-100 \%$; specificity of $85 \%-88 \%$ and accuracy of $86 \%$ $[12,13]$.Diffusion-weighted imaging (DWI) has shown to be more sensitive and specific than standard MRI It is believed to be superior for tumor detection and characterization, DWI is more sensitive than contrast enhanced CT in detecting metastases [14].CT scan has role in patients with rectal cancer, it had been reported to be an excellent method with the ability for detection tumor and demonstrate complications including perforation and obstruction that may not be clinically noticeable $[15,16]$. Some studies reported high accuracy rates for CT [17-20]but recent reports show less adequate results regarding $\mathrm{CT}$ accuracy. They noted that helical $\mathrm{CT}$ has an accuracy rates ranging between $41 \%$ and $82 \%$ [21-24], Two studies using CT scan focused on the prediction of tumor invasion $[25,26]$; another studies suggested results equal or inferior to MRI. CT is excellent for demonstrating perirectal fat and adjacent organ involvement, but it lacks accuracy for loco regional staging because of its inability to distinguish between mural layers. Histology is no longer applicable as traditional gold standard method for tumor characterization [27], and questions are still raised on the accuracy of $\mathrm{CT}$. This study was done to evaluate the CT scans for patients with rectal bleeding and suspected to have rectal neoplasm and compare their findings with those seen at endoscope and histopathology which were taken as a gold standard.

\section{Material and Methods.}

\subsection{Study Sample and Equipment used}

A sample of 50 patients, $34(68 \%)$ were males and 16 $(32 \%)$ were females with ages range between (25-85 years) ,complaining of rectal bleeding and change in bowel habits. They were clinically diagnosed as having rectal masses, all patients underwent computerized tomography and endoscopy, in addition to the histopathological examination after biopsies taken by the endoscope. The Inclusion Criteria were: Patients complaining of lower abdominal pain and rectal bleeding, both genders, Patients underwent both computerized tomography and endoscopy and patients with histopathology examination of the taken biopsies. The selected variables to be studied were: Patients age, gender, residence and rectal findings in CT, sigmoidoscopy and Biopsy. CT machine used was General electric (GE) Hi Speed NXi (dual slice) CT scanner, Manufactured 2001, Options: Advantage Workstation (AW4.0) - Reformat - Remote Tilt - Power 350mA Max. Fast scan $0.8 \mathrm{sec}$ - Extended FOV - 3.5Mhu tube - Thickness $0.5 \mathrm{~mm}$ - Helical 120 Max. Image Disk 20000 - Raw Data 1000 Max - Enhanced cooling.The Sigmoidoscope specifications were Manufacturer : karl storz, Length : $170 \mathrm{~mm}$, Outer diameter : $13 \mathrm{~mm}$, Compatible camera : karl storz endoscopy image 1,telecam, system : Dx with appropriate adapters. Equivalent illumination sources: Karl storz xenon 100, Diameter: $3.8 \mathrm{~mm}$

\subsection{Method of Examination}

\subsubsection{CT scanning and Sigmoidoscopy}

The patients were positioned on the CT examination table, lying flat on their back. Straps and pillows were used to help maintain of the correct position and to hold still during the examination. Scanning parameters were: Thickness: $5 \mathrm{~mm}$, pitch of 1.5:1, Rotation speed (RS) $=0.8 \mathrm{sec}, 2.5 \mathrm{~mm}$ reconstructions. Scout images were taken and they covered the dome of liver to symphysis pubis. Axial images were acquired from lung bases to the symphysis pubis following the administration of IV contrast medium. Intravenous contrast medium: $3-5 \mathrm{ml} / \mathrm{sec}$ for $125 \mathrm{ml}$ Omnipaque.45 second delay time. Oral contrast medium was given prior to exam. (Omnipaque 350 (undiluted) 50-100 mL, dependent on patient weight. The Sigmoidoscopy was done where the patients were placed in a left lateral position and a gentle digital rectal examination was performed. The flexible sigmoidoscope was inserted to $60 \mathrm{~cm}$, or as far as is tolerated by the patient.

\subsubsection{Methods of Evaluation}

The master data sheet was divided into : the patient gender, patient age, CT findings which mainly determine the site and character of the lesion that may be right rectal wall mass, Eccentric wall thickening, Lower third of rectum mass, Rectosigmoid tumor and Circumferential wall thickening. Sigmoidoscopy findings that involved the measured distance of the masses from the anus to above were masses detected at $2-3 \mathrm{~cm}, 4-7 \mathrm{~cm}$ and $8-15 \mathrm{~cm}$ from the anal verge, also involved the character of masses were included Stenosing tumor, Circumferential mass, Obstructive mass, Ulcerative lesion and Polypoid lesion.The histopathology results were included Moderately differentiated adenocarcinoma, Poorly differentiated adenocarcinoma, well differentiated adenocarcinoma, Tubulovillous adenoma and in some cases malignancy was not seen and the residence of patients had also been evaluated.The data were analyzed by using simple statistical graphs, mean, standard deviation, percentage, sensitivity, and accuracy using equations. TP: True Positive, TN: True negative, FN: False negative, FP: False positive: Accuracy $=\mathrm{TP}+\mathrm{TN} /$ total X 100, Sensitivity $=\mathrm{TP} / \mathrm{TP}+\mathrm{FN}$ x 100 .

\section{Results}

Table 1. Age distribution of patients with rectal neoplasms.

\begin{tabular}{lll}
\hline Age & Frequency & Percentage \\
\hline $10-25$ & 4 & $8 \%$ \\
$26-40$ & 9 & $18 \%$ \\
$41-55$ & 12 & $24 \%$ \\
$56-70$ & 18 & $36 \%$ \\
$71-85$ & 7 & $14 \%$ \\
Total & 50 & $100 \%$ \\
\hline
\end{tabular}


Table 2. The residence of the patients.

\begin{tabular}{ccc}
\hline Residence & Frequency & Percentage \\
\hline North & 3 & $6 \%$ \\
Central & 30 & $60 \%$ \\
East & 6 & $12 \%$ \\
West & 11 & $22 \%$ \\
Total & 50 & $100 \%$ \\
\hline
\end{tabular}

Table 3. Distribution of the study sample according to CT lesion character.

\begin{tabular}{lcc}
\hline CT Characterization & Frequency & Percentage \\
\hline Normal & 4 & $8 \%$ \\
Mass* & 20 & $40 \%$ \\
Tumor** & 11 & $22 \%$ \\
Circumferential Rectum wall thickening & 10 & $20 \%$ \\
Eccentric Rectum wall thickening & 5 & $10 \%$ \\
Total & 50 & $100 \%$ \\
\hline
\end{tabular}

*usage of the term (mass)the diagnosis stands for the presence of the lesions as in RT rectal wall and they were 6patients, Ampulla of rectum were (2), lower third of rectum were (12),**and The term tumor stands for the presence of the lesion were presented in the recto sigmoid region and were diagnosed in (11)cases.

Table 4. Distribution of the study sample according to Endoscopy lesion character.

\begin{tabular}{lcc}
\hline Endoscopy characterization & Frequency & Percentage \\
\hline Polyploidy mass & 8 & $16 \%$ \\
Obstructive mass & 10 & $20 \%$ \\
Ulcerative mass & 6 & $12 \%$ \\
Circumferential mass & 9 & $18 \%$ \\
Tumor & 17 & $34 \%$ \\
Total & 50 & $100 \%$ \\
\hline
\end{tabular}

Table 5. Distribution of the study sample according to Histopathology character.

\begin{tabular}{lcc}
\hline Histopathology Characterization & Frequency & Percentage \\
\hline Moderately differentiated adenocarcinoma & 22 & $44 \%$ \\
Well differentiated adenocarcinoma & 14 & $28 \%$ \\
Tubulo-villous adenoma & 4 & $8 \%$ \\
Poorly differentiated adenocarcinoma & 5 & $10 \%$ \\
No malignancy seen & 5 & $10 \%$ \\
Total & 50 & $100 \%$ \\
\hline
\end{tabular}

Table 6. CT lesion character \& Lesion locations mean Distance from anus P-value 0.001 .

\begin{tabular}{lc}
\hline CT (site) & Mean \pm S D \\
\hline Normal & $11.5 \pm 1.0$ \\
Mass & $6.4 \pm 4.1$ \\
Tumor & $11.1 \pm 1.9$ \\
Circumferential wall thickening & $6.4 \pm 3.5$ \\
Eccentric thickening & $4.8 \pm 2.6$ \\
Total & $7.6 \pm 4.0$ \\
\hline
\end{tabular}

Values are expressed as Mean $\pm \mathrm{SD} *$ Normal $\mathrm{p}$-value is $<0.05$; therefore the statistical difference is highly significant
Table 7. Endoscopy Characterization \& Lesion locations mean Distance from anus $P$-value 0.038 .

\begin{tabular}{cc}
\hline Endoscopy & Mean \pm S D \\
\hline Polyploidy & $10.9 \pm 3.2$ \\
Obstructive & $8.0 \pm 3.9$ \\
Ulcerative & $8.5 \pm 3.9$ \\
Circumferential & $7.3 \pm 3.6$ \\
Tumor & $5.7 \pm 3.9$ \\
Total & $7.6 \pm 4.0$ \\
\hline
\end{tabular}

Values are expressed as Mean $\pm \mathrm{SD} *$ Normal $\mathrm{p}$-value is $<0.05$; therefore the statistical difference is highly significant

Table 8. Biopsy Results of lesions characters \& Lesion locations mean Distance from anus $(P$-value $=0.161)$.

\begin{tabular}{lc}
\hline \multicolumn{1}{c}{ Biopsy } & Mean \pm S D \\
\hline Moderately differentiated adenocarcinoma & $7.2 \pm 4.1$ \\
Well differentiated adenocarcinoma & $7.7 \pm 3.9$ \\
Tubulovillous adenoma & $8.3 \pm 4.5$ \\
Poorly differentiated adenocarcinoma & $5.0 \pm 4.1$ \\
No malignancy seen & $11.2 \pm 1.1$ \\
Total & $7.6 \pm 4.0$ \\
\hline
\end{tabular}

Values are expressed as Mean \pm SD

Table 9. Accuracies of CT, Endoscopy, and Biopsy in 50 patients.

\begin{tabular}{cccc}
\hline Parameter & CT & Endoscopy & Biopsy \\
\hline Sensitivity (\%) & $69.6 \%$ & $88.6 \%$ & $89.1 \%$ \\
Specificity (\%) & $100.0 \%$ & $66.7 \%$ & $100.0 \%$ \\
Accuracy (\%) & $72.0 \%$ & $86.0 \%$ & $90.0 \%$ \\
\hline
\end{tabular}

\section{Discussion}

The researchers used three methods to localize and characterize the rectal neoplasms ;including CT, endoscopy and biopsy, and studied four variables including patient's age, gender, residence and distance from anus.

To study the variables that diagnose the rectal neoplasms; ANOVA independent methods, mean, standard deviation and $p$-value were used. The maximum affected age were the age between 56-70 years representing 18 out of $50(36 \%)$ of samples and the minimum affected age were the ages between $10-25$ years $(8 \%)$ of the samples, ages between (26-40 years) constituting $18 \%$ while (41-55 years) representing $24 \%$ and (71-85 years) $14 \%$ table[1] the results reflected that the possibility of the occurrence of the rectal neoplasms, increased with age this is compatible to what was mentioned by the National Cancer Institute which stated that the incidence rate of colorectal cancer is more than 15 times higher in adults 50 years and older than in those 20 to 49 years. [28].

The study showed the gender incidence of patients with rectal neoplasms were detected in male patients by 
$34(68 \%)$, while the females by $16(32 \%)$. Other similar studies showed that colorectal cancer incidences are higher in men than in women; the reasons are not completely understood, but this may reflect the relation between gender hormones and risk pattern. [29].Table [2] showed the residence of patients in Sudan with rectal neoplasm. The most cases were from Central state, it represent $30(60 \%)$ of the cases, this may be similar to was mentioned by the American Cancer Society which stated that the causative factors which may affect the distribution of rectal neoplasm included regional variations in risk factors which are influenced by socioeconomic factors and medical services.[30]Different sites of the rectal neoplasm were detected by CT. Rectal lesions which were characterized as masses; were detected at area of the lower third of rectum constituting $22(44 \%)$ of cases, while the other cases were described as circumferential rectal wall thickening representing $10(20 \%)$ cases, eccentric thickening $4(8 \%)$, recto sigmoid tumor $10(20 \%)$ and the rectum was normal in $4(8 \%)$ of the samples. Table [3] presented the findings; in addition $\mathrm{CT}$ scanning showed no lymph node involvement or any metastatic changes in all the cases.

The masses detected by endoscopy were characterized as polyploidy $8(16 \%)$,obstructive $10(20 \%)$, ulcerative $6(12 \%)$,circumferential $19(18 \%)$ and toumers $17(34 \%)$ as presented in table [4] The lesions were localized according to the distance from anus verge; all the polyploid masses were found from a distance of $10.9 \pm 3.2$ where the obstructive masses were found at $8.0 \pm 3.9$, ulcerative masses at $8.5 \pm 3.9$, circumferential masses at $7.3 \pm$ 3.6,tumors at $5.7 \pm 3.9$.

There are significant relation between the character and location of the masses when diagnosed by endoscope at $P$-value 0.038 as shown in table [7], which means we can predict the lesions characters according to their site. Studies showed that a percentages of around $30 \%-40 \%$ of colorectal cancer was defined to arise from the rectum which is defined as the distal margin of tumor within $15 \mathrm{~cm}$ of the anal verge $[31,32]$.The importance of screening is to prevent colorectal cancer because most colorectal cancers develop from adenomatous polyp [30] .The tumor typically begins as a non cancerous polyp, adenomatous polyps or adenomas and may progress to cancer.[33] About 96\% of colorectal cancers are adenocarcinomas, which evolve from glandular tissue.[34]Colorectal cancer screening by colonoscopy is highly sensitive because of its ability to monitor entire colon; diagnose lesions, and removal of polyps if present in the same investigation session. It has been estimated that colonoscopy screening can prevent about $65 \%$ of colorectal cancer cases.[35, 36].

The study can predict the tumor type according to its location away from anus verge by CT because there are a significant relation noticed between the CT diagnoses and citation of the lesion from anus at $p$ value 0.001 . Masses were found at distance $6.4 \pm 4.1 \mathrm{~cm}$ from anus verge, enhanced tumors were located at11.1 $\pm 1.9 \mathrm{~cm}$, circumferential rectal wall thickening at $6.4 \pm 3.5 \mathrm{~cm}$ and the eccentric thickening were at $4.8 \pm 2.6 \mathrm{~cm}$ as seen in table [6]

The histopathological result of the lesions were classified as moderately differentiated adenocarcinoma22(44\%) of total cases, while the minority of cases were diagnosed as Tubulovillous adenoma 4(8\%)of cases. Other biopsies were classified as poorly differentiated adenocarcinoma $5(10 \%)$ of samples, well differentiated adenocarcinoma 14(28\%) and malignancies were not seen in $5(10 \%)$ of the biopsies table [5].

No significant relation was found between the biopsy results and the presence of the lesions through distance from anus at $P$-value 0.161 as seen in table [8].

The value of CT and colonoscopy in characterizing and localizing rectal tumors were presented. The study showed that they can localize the lesions significantly better than the biopsy which is an invasive technique .The accuracy and sensitivity of the CT as related to the Endoscopy and biopsy result was studied and represented in table [9].Studies mentioned that Colonoscopy and biopsy is considered as the gold standard investigations to confirm the diagnosis of rectal cancer and to exclude other lesions. [30]The prediction of rectal cancer is directly related to extramural tumor spread into the meso-rectum [37] colonoscopy cannot study the invasion of the lesion which makes the diagnosis dependency of less accuracy.

Rectal cancer diagnosis can be confused with inflammation, which can also lead to over- staging [38] .Therefore the role of CT scan will be better as it can characterize the lesion with better contrast enhancement and localize the lesions with exhibition to other organs involvement.

Table [10] showed different values for CT accuracy, sensitivity and specificity, The reduction of CT accuracy, sensitivity, specificity in our study may be due to variation in data presentation as sample size or the type of CT scanner similar to what was mentioned in other similar studies[39] another studies suggested that the accuracy of CT for prediction of the circumferential resection margin in tumors is high in the proximal and middle rectum but of less accuracy in the distal rectum due to low contrast resolution and the complicated anatomy of that region[40].

Because the early diagnoses of rectal cancer may improve the disease prognosis and treatment outcome, this study recommend that CT should be done together with sigmoidoscopy to give a clear description of the lesion character, citation from anus verge as well as other organs involvement which may help in proper diagnoses.

Biopsy must be taken from each mass and sent to histopathological investigation to determine the nature of the masses and confirm the results. The cyclic investigations of rectum are recommended for patients with ages above 50 years old as well as patients with rectal bleeding using CT scan and sigmoidoscopy. 
Table 10. Similar studies about the Accuracies of CT in diagnosis of rectal neoplasm.

\begin{tabular}{lllll}
\hline \multicolumn{1}{c}{ Study } & \multicolumn{1}{c}{ Accuracy } & \multicolumn{1}{c}{ sensitivity } & \multicolumn{1}{c}{ specificity } & \multicolumn{1}{c}{ item } \\
\hline Martellucci J et al[28] & $79 \%$ & - & - & Preoperative Radio Chemotherapy In Advanced Rectal Cancer \\
Beynon J. et al[29] & $82 \%$ & $86 \%$ & $62 \%$ & Operative Assessment Of Local Invasion In Rectal Cancer \\
& $72.4 \%$ & $69.0 \%$ & $88.7 \%$ & \\
Ozel B et al[30] & $83.6 \%$ & $65.5 \%$ & $84.5 \%$ & Detection Of Large Colorectal Polyps And Cancer \\
& $80.3 \%$ & $82.8 \%$ & $77.5 \%$ & \\
Perry J. Pickhardt et al[31] & - & $96.1 \%$ & - & Cancer Detection \\
Halligan S et al[32] & $73 \%, 98 \%$ & $93 \%, 96 \%$ & $97 \%, 95 \%$ & Detection Of Colorectal Polyps And Cancer \\
MSAC (2006)[33] & - & $97 \%$ & $98 \%$ & Cancer Detection \\
Purkayastha et al[34] & - & $95 \%$ & $95 \%$ & Diagnosis Of Colorectal Cancer \\
\hline
\end{tabular}

\section{Acknowledgements}

Our Thanks are extended to Sudan University of Science and Technology, College Of Medical Radiological Science and Soba Hospital-Khartoum-Sudan.

\section{References}

[1] Z.R. Jass,. Pathological prognostic indicators in rectal cancer. Hum Pathol; 199425:498-505.

[2] J.R .Jass. Prognostic value of direct spread in Dukes' C cases of rectal cancer,. Dis Colon Rectum; 1989,32:477-480.

[3] R. Tang, J.Y .Wang, J.S. Chen, et al.,. Survival impact of lymph node metastasis in TNM stage III carcinoma of the colon and rectum. J Am Coll Surg1995; 180: 705-712.

[4] C.G .Willett, K. Badizadegan, M. Ancukiewicz, P.C. Shellito.,. Prognostic factors in stage T3N0 rectal cancer: do all patients require postoperative pelvic irradiation and chemotherapy? Dis Colon Rectum1999; 42:167-173.

[5] N .Wolmark, B .Fisher, H.S. Wieand., The prognostic value of the modifications of the Dukes' C class of colorectal cancer. Ann Surg 1986; 203:115-122.

[6] D.C. Rockey, E .Paulson, D .Niedzwiecki, et al.Analysis of air contrast barium enema, computed tomographic colonography, and colonoscopy: prospective comparison. Lancet. 2005; 365(9456):305-311.

[7] B .Levin, D.A. Lieberman, B .McFarland, et al.Screening and surveillance for the early detection of colorectal cancer and adenomatous a Polyps: joint guideline from the American Cancer Society, the US Multi-Society Task Force on Colorectal Cancer, and the American College of Radiology. CA Cancer J Clin2008.;58(3):130-160

[8] G.A Boyce, M.VSivak, I.C Lavery, et al.Endoscopic ultrasound in the pre-operative staging of rectal carcinoma. Gastrointest Endosc1992; 38(4):468-471

[9] U. Herzog, M .Von Flue, P .Tondelli, J.P Schuppisser.How accurate is endorectal ultrasound in the preoperative staging of rectal cancer? Dis Colon Rectum; 199336(2):127-134

[10] A.Waizer, S .Zitron, D. Ben-Baruch, J. Baniel, Y. Wolloch, M .Dintsman..Comparative study for preoperative staging of rectal cancer. Dis Colon Rectum1989; 32(1):53-56
[11] K.H Rotte, L. Kluhs, H. Kleinau, E.. Kriedemann,.Computed tomography and endosonography in the preoperative staging of rectal carcinoma. Eur J Radiol1989; 9(3):187-190

[12] P .Videhult, K. Smith, P .Lundin, W. Kraaz.Magnetic resonance imaging for preoperative staging of rectal cancer in clinical practice: high accuracy in predicting circumferential margin with clinical benefit. Colorectal Dis2007; 9(5):412-419.

[13] S .Purkayastha, P.P. Tekkis, T. Athanasiou, H.S .Tilney, A.W Darzi, A.G .Heriot.. Diagnostic precision of magnetic resonance imaging for preoperative prediction of the circumferential margin involvement in patients with rectal cancer. Colorectal Dis2007;9(5):402-411

[14] R.Sugita,K.Ito,N.Fujita, S.Takahashi.Diffusion-weighted MRI in abdominal oncology: clinical applications. World J Gastroenterol2010; 16(7):832-836

[15] R.F .Thoeni..Colorectal cancer. Radiologic staging. Radiol Clin North Am1997; 35(2):457-485

[16] R. Farouk, H .Nelson, E. Radice, S .Mercill, Gunderson L. Accuracy of computed tomography in determining resectability for locally advanced primary or recurrent colorectal cancers. Am J Surg1998; 175(4):283-287

[17] R. F. Thoeni, A.A.Moss, P. Schnyder, A. R. Margulis. Detection and staging of primary rectal and recto sigmoid cancer by computed tomography. Radiology 1981; 141: 135-138

[18] W.M. Thompson, R.A .Halvorsen, W.L .Foster Jr,Roberts L, R. Gibbons..Preoperative and postoperative CT staging of recto sigmoid carcinoma.AJR1986;146:703-710

[19] Freeny PC, Marks WM, Ryan JA.1986. Colorectal carcinoma evaluation with CT: preoperative staging and detection of postoperative recurrence. Radiology;158:347-353

[20] A .Maier, M. Fuchsjäger..Preoperative staging of rectal cancer. Eur J Radiol2003;47:89-97

[21] Thoeni RF. 1997.Colorectal cancer: radiological staging. Radiol Clin N Am;35:457-458

[22] G .Angelelli, L .Macarini, L. Lupo.Rectal carcinoma :CT staging with water as contrast medium. Radiology 1990; 177: $511-514$

[23] Hundt W, Braunschweig R, Reiser M. 1999.Evaluation of spiral CT in staging of colon and rectum carcinoma. Eur $\mathrm{J}$ Radiol;9:78-84 
[24] M .Chiesura-Corona, P.C. Muzzio, G. Giust, Zuliani , S Pucciarelli, P. Toppan. Rectal cancer:.CT local staging with histopathology correlation. Abdom Imaging2001;26:134-138

[25] A .Taylor, A .Slater, N .Mapstone, et al. ,. Staging rectal cancer: MRI compared to MDCT. Abdom Imaging 2007,32:323-327

[26] S .Wolberink, R.G .Beets-Tan, D .de Haas-Kock, et al.,.Conventional $\mathrm{CT}$ for the prediction of an involved circumferential resection margin in primary rectal cancer. Dig Dis 2007,25:80-85

[27] Roy Vliegen, Raphaela Dresen, Geerard Beets, Alette Daniels-Gooszen, Alfons Kessels, Jos van Engelshoven, Regina Beets-Tan. ,.The accuracy of Multi-detector row CT for the assessment of tumor invasion of the meso-rectal fascia in primary rectal cancer,Abdom Imaging 2008,33:604-610

[28] SEER Stat Database: Incidence - SEER 17 Regs Limited-Use + Hurricane Katrina Impacted Louisiana Cases, Nov 2009 Sub (2000-2007)Katrina/Rita Population AdjustmentLinked To County Attributes - Total U.S., 1969-2007 Counties [computer program]: Surveillance, Epidemiology, and End Results (SEER) Program (www.seer.cancer.gov), National Cancer Institute, DCCPS, Surveillance Research Program, Cancer Statistics Branch, released April 2010, based on the November 2009 submission

[29] G .Murphy, S.S. Devesa, A.J .Cross, P.D Inskip, K.A McGlynn, M.B Cook.Sex disparities in colorectal cancer incidence by anatomic sub site, race and age. Int $\mathrm{J}$ Cancer;2010

[30] American Cancer Society. Cancer Prevention \& Early Detection Facts \& Figures 2010. Atlanta, GA: American Cancer Society; 2010.

[31] D. Hayne, R.S .Brown, M .McCormack, M.J .Quinn, H.A Payne, P .Babb..Current trends in colorectal cancer: site, incidence, mortality and survival in England and Wales. Clin Oncol (RColl Radiol) 2001; 13: 448-452
[32] G .Salerno, I.R .Daniels, B.J .Moran, A .Wotherspoon, G. Brown. Clarifying margins in the multidisciplinary management of rectal cancer: the MERCURY experience. Clin Radiol 2006; 61:916-923

[33] Levine JS, Ahnen DJ. 2006. Clinical practice. Adenomatous polyps of the colon. N Engl J ed.; 355(24):2551-2557

[34] S.L .Stewart, J.M. Wike, I .Kato, D.R. Lewis, F .Michaud..A population-based study of colorectal cancer histology in the United States, 1998-2001. Cancer.;107(5 Suppl): 2006; $1128-1141$

[35] H .Brenner, J .Chang-Claude, C.M .Seiler, T .Sturmer, M .Hoffmeister.Potential for colorectal cancer prevention of sigmoidoscopy versus colonoscopy: population-based case control study. Cancer Epidemiol Biomarkers Prev.; 2007;16(3):494-499

[36] C.J .Kahi, Imperiale TF, Juliar BE, Rex DK.Effect of screening colonoscopy on colorectal cancer incidence and mortality. Clin Gastroenterol Hepatol. 2009; 7(7):770-775

[37] S.J. Cawthorn, D.V. Parums, N.M .Gibbs, et al. Extent of mesorectal spread and involvement of lateral resection margins as prognostic factors after surgery for rectal cancer. Lancet 1990; 335:1055-1059

[38] G .Low, L.M .Tho, E .Leen, et al.The role of imaging in the preoperative staging and post-operative follow-up of rectal cancer. Surgeon2008; 6(4):222-231

[39] Shandra Bipat, S .Afina. Glas, J. M Frederik. Slors, Aeilko H. Zwinderman, M. M. Patrick. Bossuyt, Jaap Stoker, .Rectal Cancer: Local Staging and Assessment of Lymph Node Involvement with Endoluminal US, CT, and MR Imaging-A Meta-Analysis1 , Radiology Volume 232, Number 2004;3:773-783

[40] G .Panzironi, M .De Vargas Macciucca, L. Manganaro, et al.: Preoperative loco regional staging of rectal carcinoma: comparison of MR, TRUS and Multislice CT. Personal experience. Radiol Med (Torino2004); 107:344-355 